This is a postprint version of the following published document:

Alonso-Pardo, J.; Sánchez-Arriaga, G. Kite model with bridle control for wind-power generation, in: Journal of Aircraft, Vol. 52, No. 3 (2015), pp. 917-923.

DOI: https://doi.org/10.2514/1.C033283

(C) 2015 by the American Institute of Aeronautics and Astronautics, Inc. All rights reserved. 


\title{
Kite Model with Bridle Control for Wind-Power Generation
}

\author{
J. Alonso-Pardo* and G. Sánchez-Arriaga \\ Universidad Politécnica de Madrid, 28040 Madrid, Spain
}

DOI: $10.2514 / 1 . C 033283$

\begin{abstract}
A compact flight dynamics model of a kite is developed by using Lagrangian formulation. The lengths of the three ropes of the bridle and the tether of the kite depend on time and are used to implement an open-loop control scheme of the kite system. By imposing simple time-periodic control laws, two pumping strategies for wind-energy generation are explored. Periodic trajectories of the system and their stability properties (Floquet characteristic multipliers) are computed numerically. As the amplitudes of the figure-eight paths are increased, the system becomes more efficient but less stable. A cyclic-fold bifurcation is detected for a very large lateral displacement of the kite. The impact of the control-law parameters on the generated power, including the period and the amplitude, is investigated. The results indicate that a correct design of the control could provide an optimal energy-generation system and a robust scheme to exploit high-altitude winds.
\end{abstract}

$\begin{array}{lll} & \text { Nomenclature } \\ C_{l p}, C_{m q}, C_{n r} & =\text { aerodynamic torque coefficients, } \mathrm{s} / \mathrm{rad} \\ C_{m \alpha}, C_{l \beta}, C_{n \beta}, & =\text { aerodynamic torque coefficients } \\ C_{X \alpha}, C_{Y \beta}, C_{Z \alpha} & =\text { aerodynamic force coefficients, } 1 / \mathrm{rad} \\ C_{X 0}, C_{Z 0}, C_{m 0} & =\text { aerodynamic coefficients } \\ c & =\text { kite chord, } \mathrm{m} \\ \boldsymbol{F}_{A} & =\text { aerodynamic force, } \mathrm{N} \\ \boldsymbol{F}_{T} & =\text { total tension force, } \mathrm{N} \\ g & =\text { gravitational acceleration, } \mathrm{m} / \mathrm{s}^{2} \\ h & =\text { kite semiminor axis, } \mathrm{m} \\ L & =\text { tether length, } \mathrm{m} \\ l & =\text { kridle length, } \mathrm{m} \\ M & =\text { angular-velocity components, rad } / \mathrm{s} \\ p, q, r & =\text { aerodynamic torque, } \mathrm{N} \cdot \mathrm{m} \\ \boldsymbol{T} & =\text { aerodynamic velocity, } \mathrm{m} / \mathrm{s} \\ V_{A} & =\text { reference velocity, } \mathrm{m} / \mathrm{s} \\ V_{T} & =\text { useful electrical power, } \mathrm{W} \\ \dot{W}_{\text {ele }} & =\text { Eody frame of reference } \\ X_{B}, Y_{B}, Z_{B} & =\text { angle of attack, rad } \\ X_{E}, Y_{E}, Z_{E} & =\text { slipside angle, rad } \\ \alpha & =\text { tether elevation angle, rad } \\ \beta & =\text { bridle elevation angle, rad } \\ \Gamma & =\text { bridle lateral angle, rad } \\ \delta & =\text { pitch angle, rad } \\ \eta & =\text { air density, kg/m }{ }^{3} \\ \theta & =\text { roll angle, rad } \\ \rho_{0} & = \\ \phi & & \text { yaw angle, rad } \\ \varphi & & \\ \psi & & \end{array}$

\section{Introduction}

M OST of wind-energy-production systems, like wind turbines, extract power at low altitudes. Although it has made possible an extensive wind exploitation and a remarkable growth in later years,

Received 18 November 2014; revision received 17 February 2015; accepted for publication 19 February 2015; published online XX epubMonth XXXX. Copyright (c) 2015 by the American Institute of Aeronautics and Astronautics, Inc. All rights reserved. Copies of this paper may be made for personal or internal use, on condition that the copier pay the $\$ 10.00$ per-copy fee to the Copyright Clearance Center, Inc., 222 Rosewood Drive, Danvers, MA 01923; include the code 1533-3868/YY and $\$ 10.00$ in correspondence with the CCC.

*Aerospace Engineer, Department of Applied Physics, Escuela Técnica Superior de Ingeniería Aeronáutica y del Espacio.

${ }^{\dagger}$ Assistant Professor, Department of Applied Physics, Escuela Tecnica Superior de Ingeniería Aeronáutica y del Espacio; also Visiting Professor (Posdoc), Department of Bioengineering and Aerospace Engineering, Universidad Carlos III de Madrid, 28911 Madrid, Spain. important benefits would be obtained if power systems were operated at high altitudes. Winds increase with height, and for the jet streams, located between 7 and $16 \mathrm{~km}$ of altitude, wind speeds are an order of magnitude faster than those near the ground [1]. In addition, the substitution of wind turbines by lightweight structures placed at a high altitude, like kites, would reduce the costs and the visual impact of these installations. Therefore, new opportunities will appear if high-altitude wind-production systems (HAWPS) are developed and tested. If successful, they will probably require restricted zones in air traffic (as already exist for other power-generation facilities like nuclear plants).

Previous work showed several possibilities in the designs of HAWPS and also real demonstrations of technology and prototypes. They included FlyGen concepts, which generate the power onboard, and GroundGen concepts that use the traction force to obtain electrical energy by using a tether connected to a generator on the ground. Among the GroundGen schemes, one finds the crosswind kite power [2], the Laddermill [3], the pumping mill [4], and kites operating in pumping modes $[5, \overline{6}]$. The latter is based on a periodic cycle made up of two different phases. During the reel-out phase, the traction force in the tether, generated by the kite flying in a crosswind maneuver, is used to produce electrical energy by spinning a generator at ground. The tether is winched in, and the kite flies back during the reel-in phase.

The dynamics and control of the kite are two relevant difficulties of GroundGen concepts. They involve the well-known characteristics of any aircraft, a highly flexible structure, and also constraints imposed by the tether and the bridle lines. The latter, which are only active if the ropes are under tension, are coupled with the dynamics of the ropes. On the top of this, the kite should also operate in changing wind conditions. These difficulties were recognized by the community that carried out theoretical analyses of the kite problem. Pioneering work on the stability of towed bodies [7-9] have been followed by several analyses of the longitudinal [10] and the lateraldirectional $[11,12]$ stability of the kite. Flexibility effects were also addressed [13]. To achieve optimal energy pumping maneuvers, closed-loop schemes have been proposed in the past [14-18]. The result is a robust system that is able to follow a certain prescribed trajectory. The control of these schemes, however, needs to be fed by instruments or sensors that measure the state of the kite. This increases the complexity of the system. An alternative strategy, explored in this work, is an open-loop control without sensors. In this case, the stability of the orbit plays a critical role; because the kite should fly following a periodic trajectory, the control laws must be designed carefully to yield a stable periodic orbit. In order to achieve optimal energy pumping manoeuvres, closed-loop schemes have been proposed in the past [14-19].

This work presents a dynamic model of a kite with a bridle made of three lines. This model, which explicitly eliminates the constraint forces, extends the one presented in $[10,12]$ in several directions. As shown in Sec. III, constraint forces and the generated electrical 
energy are found once the kite trajectory is known. The computation of stable periodic orbits and the airborne energy generation with both a pumping mill strategy and a crosswind pumping scheme are presented in Sec. IV. The main results of the work and a discussion about the application of the model for wind-power generation are presented in Sec. V.

\section{Kite-System Model}

This section closely follows [12], in which a kite model, including lateral dynamics, was presented. The main extensions to this work are 1) inertia kite model with a semi-elliptical cross section, 2) aerodynamic model with drag along the longitudinal kite axis, and 3) time-dependent tether and bridle lines lenghts given by external control laws. These three novelties are here described in detail. (See [12] for a thorough revision of other parts of the model.) For convenience, we will use the notation $s \alpha$ and $c \alpha$ to denote the trigonometric functions $\sin \alpha$ and $\cos \alpha$, respectively.

The kite, here taken as a rigid solid, has a semi-elliptical cross section of semiminor axis equal to $h$, major axis $b$ (wingspan), and chord $c$. For inertial considerations, kite thickness is neglected and its areal density, $\sigma \equiv m / A$, is assumed constant. Here, $M$ and $A$ are the mass and the surface of the kite, respectively. The center of mass $G$ of the kite is placed at the intersection of the two planes of symmetry of the kite and at distance $h_{G}$ from a plane normal to the semi-elliptical cross section that passes through the focal points. The analytical expressions for $A, h_{G}$, and the inertial tensor $\vec{I}_{G}$ as a function of $h, b$, $c$, and $\sigma$ are given in Appendix A1.

The bridle is made up of three lines that connect the points $A, B$, and $C$ of the kite with the tether at the frictionless point $Q$, respectively (see Fig. 1b). We remark that three is the maximum number of bridle lines that can connect the kite with point $Q$ without making the problem hyperstatic. At point $Q$, there is a control unit with a motor that controls the lengths of the bridle lines. At the ground attachment point $O$, there is a drum/generator module that is used to extract electrical energy from the kite system or reel in the tether. The bridle lines and the tether are here assumed as straight segments with lengths given by certain time-dependent control laws. Because these lengths are known functions, the kite system has five degrees of freedom that we organized in the state vector:

$$
\boldsymbol{x}_{s}=\left[\begin{array}{lllll}
\Gamma & \theta & \varphi & \psi & \phi
\end{array}\right]^{T}
$$

in which $\Gamma$ and $\varphi$ are the elevation and lateral angles of the tether, respectively, and $\theta, \phi$, and $\psi$ are the pitch, roll, and yaw angles of the kite, respectively (see Fig. 1).

Instead of the lengths of the bridle $(|\overleftarrow{A Q}|,|\overleftarrow{B Q}|$, and $|\overleftarrow{C Q}|)$, our model uses the normalized length $\tilde{l}(t) \equiv|\overleftarrow{Q G}| / L_{0}$ and the angles $\delta(t)$ and $\eta(t)$ (see Fig. 1b), which yield to a more compact formulation of the equations. Here, $L_{0}$ is a constant reference length. These
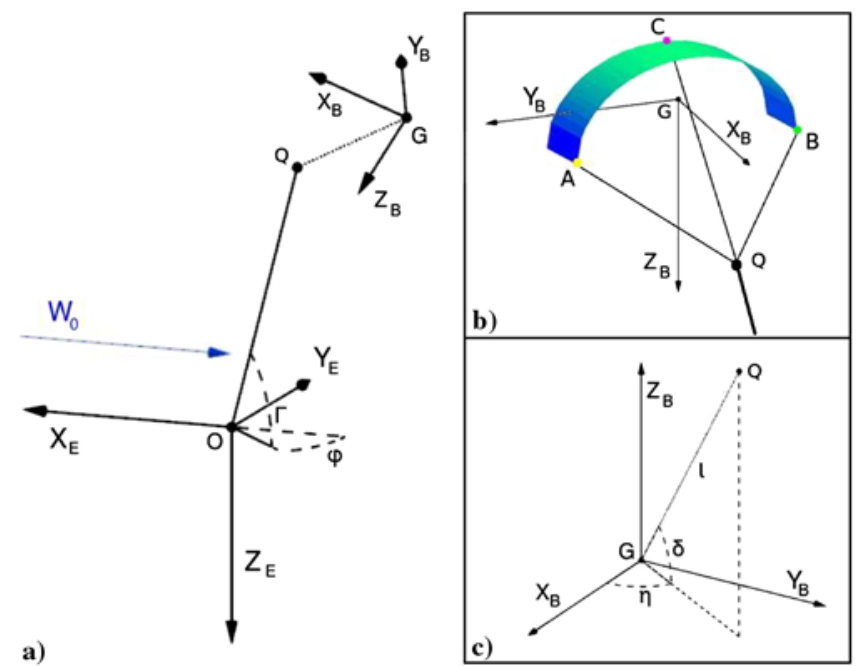

Fig. 1 Frames of reference and kite configuration. dimensionless parameters, together with the normalized tether length, $\tilde{L}(t) \equiv|\overleftarrow{O Q}| / L_{0}$, form the control vector of the model:

$$
\boldsymbol{x}_{c}=\left[\begin{array}{llll}
\tilde{L} & \tilde{l} & \delta & \eta
\end{array}\right]^{T}
$$

Because bridle lengths are important for kite design, the analytical relations between the bridle lengths and the control variables are given in Appendix A2.

An Earth-fixed inertial frame $S_{E}$ with origin at the ground attachment point $O, z_{E}$ axis along the vertical, and the wind velocity $\boldsymbol{W}_{0}$ contained in the $x_{E}-z_{E}$ plane is used. A second frame of reference $S_{B}$ with origin at the center of mass $G$ of the kite, attached to it, and axis equal to the principal axis of inertia relative to $G$ is also introduced (see Fig. 1). The Earth and body frames have vector bases $\left(\boldsymbol{i}_{E}, \boldsymbol{j}_{E}, \boldsymbol{k}_{E}\right)$ and $\left(\boldsymbol{i}_{B}, \overline{\boldsymbol{j}}_{B}, \boldsymbol{k}_{B}\right)$, respectively. The components of a vector in these bases are related by the matrix $\overline{\boldsymbol{R}}$, which contains the kite pith, roll, and yaw $(\theta, \phi, \psi)$ angles (see Appendix $\underline{\mathrm{A} 3}$ ). The angular velocity of the kite (or the body frame), $\boldsymbol{\omega} \equiv p \boldsymbol{i}_{B}+q \boldsymbol{j}_{B}+r \boldsymbol{k}_{B}$, is given by

$$
\boldsymbol{\omega} \equiv \sqrt{\frac{g}{L_{0}}} \tilde{\boldsymbol{\omega}}=\sqrt{\frac{g}{L_{0}}} \overline{\mathbf{\Omega}} \cdot \dot{\boldsymbol{x}}_{s}
$$

in which $\overline{\boldsymbol{\Omega}}$ is a dimensionless tensor given in Appendix A4, and the dot denotes a derivative with respect to the $\overline{\text { normalized }}$ time $\tau \equiv t / \sqrt{L_{0} / g}$.

\section{A. Kinetic and Potential Energies}

Lagrange equations involve some tedious but straightforward calculations concerning the kinematics of the kite, its potential and kinetic energies, and the generalized forces. Here, we summarize some auxiliary calculations and intermediate steps. The vector position of the center of mass of the kite, $\boldsymbol{r}_{G}=\overleftarrow{O Q}+\overleftarrow{Q G}$, is

$$
\begin{aligned}
\frac{\boldsymbol{r}_{G}\left(\boldsymbol{x}_{s}, t\right)}{L_{0}}= & -\tilde{L}\left[c \Gamma\left(c \varphi \boldsymbol{i}_{E}+s \varphi \dot{j}_{E}\right)+s \Gamma \boldsymbol{k}_{E}\right] \\
& -\tilde{l}\left[c \delta\left(c \eta \boldsymbol{i}_{B}+s \eta \boldsymbol{j}_{B}\right)+s \delta \boldsymbol{k}_{B}\right]
\end{aligned}
$$

The system is holonomic (integrable constraints) and rheonomic because time explicitly appears in Eq. (4) due to the control laws $\boldsymbol{x}_{c}(t)$.

The velocity vector of the center of mass $\boldsymbol{v}_{G}=\left(\mathrm{d} \boldsymbol{r}_{G} / \mathrm{d} t\right)_{S_{E}}$ is found with the help of the Coriolis theorem $\boldsymbol{v}_{G}=$ $(\mathrm{d} \overleftarrow{O Q} / \mathrm{d} t)_{S_{E}}=(\mathrm{d} \overleftarrow{Q G} / \mathrm{d} t)_{S_{B}}+\omega \times \overleftarrow{Q G}$. The velocity vector in the Earth frame is

$$
\boldsymbol{v}_{G} \equiv \sqrt{g L_{0}} \tilde{\boldsymbol{v}}_{G}=\sqrt{g L_{0}}\left(\overline{\boldsymbol{S}} \dot{\boldsymbol{x}}_{s}+\overline{\boldsymbol{C}} \dot{\boldsymbol{x}}_{c}\right)
$$

in which the matrices $\overline{\boldsymbol{S}}$ and $\overline{\boldsymbol{C}}$, which depend on $\boldsymbol{x}_{s}$ and $\boldsymbol{x}_{c}$, are given in Appendix A4.

The kinetic energy of the kite, $E_{k}=$ $1 / 2\left(m \boldsymbol{v}_{G}^{T} \cdot \boldsymbol{v}_{G}\right)+1 / 2\left(\boldsymbol{\omega}^{T} \cdot \overline{\boldsymbol{I}}_{G} \cdot \boldsymbol{\omega}\right)$, is written in terms of the state and control vectors. Using Eqs. (3) and ()ㅡㄹ yields

$$
\begin{aligned}
E_{k} & \equiv \frac{1}{2} M L_{0}^{2} \tilde{E}_{k} \\
& =\frac{1}{2} M L_{0}^{2}\left[\dot{\boldsymbol{x}}_{s}^{T} \cdot \overline{\boldsymbol{M}}_{s} \cdot \dot{\boldsymbol{x}}_{s}+2 \dot{\boldsymbol{x}}_{s}^{T} \cdot \overline{\boldsymbol{M}}_{s c} \cdot \dot{\boldsymbol{x}}_{c}+\dot{\boldsymbol{x}}_{c}^{T} \cdot \overline{\boldsymbol{M}}_{c} \cdot \dot{\boldsymbol{x}}_{c}\right]
\end{aligned}
$$

in which $\overline{\boldsymbol{M}}_{s} \equiv \overline{\boldsymbol{S}}^{T} \cdot \overline{\boldsymbol{S}}+\overline{\boldsymbol{\Omega}}^{T} \cdot \tilde{\boldsymbol{I}}_{G} \cdot \overline{\boldsymbol{\Omega}}, \overline{\boldsymbol{M}}_{s c} \equiv \overline{\boldsymbol{S}}^{T} \cdot \overline{\boldsymbol{C}}$, and $\overline{\boldsymbol{M}}_{c} \equiv$ $\overline{\boldsymbol{C}}^{T} \cdot \overline{\boldsymbol{C}}$. Here, $\tilde{\boldsymbol{I}}_{G} \equiv \overline{\boldsymbol{I}}_{G} / M L_{0}^{2}$ is the dimensionless inertial tensor about $G$ (see Appendix A1).

On the other hand, with the aid of Eq. (4), one finds the gravitational potential energy of the kite:

$$
\begin{aligned}
U\left(\boldsymbol{x}_{s}, \boldsymbol{x}_{c}\right) & \equiv M g L_{0} \tilde{\mathcal{U}} \\
& =M g L_{0}\{\tilde{L} s \Gamma+\tilde{l}[(c \delta s \eta s \phi+s \delta c \phi) c \theta-c \delta c \eta s \theta]\}
\end{aligned}
$$




\section{B. Wind Velocity and Aerodynamic Forces}

The model considers a time-dependent wind velocity contained in the $x_{E}-z_{E}$ plane, $\boldsymbol{V}_{w}(t)=-\sqrt{g L_{0}} \tilde{V}_{w}(\tau) \boldsymbol{i}_{E}$. The aerodynamic velocity of the kite is then given by $\boldsymbol{V}_{A}=\boldsymbol{v}_{G}-\boldsymbol{V}_{w}$, and its components in the Earth frame are

$$
\boldsymbol{V}_{A} \equiv \sqrt{g L_{0}} \tilde{\boldsymbol{V}}_{A}=\sqrt{g L_{0}}\left[\overline{\boldsymbol{S}} \dot{\boldsymbol{x}}_{s}+\overline{\boldsymbol{C}} \dot{\boldsymbol{x}}_{c}+\tilde{V}_{w}(\tau) \boldsymbol{i}_{E}\right]
$$

The aerodynamic force $\left(\boldsymbol{F}_{A} \equiv M g \tilde{\boldsymbol{F}}_{A}\right)$ and its torque about the center of mass of the kite $\left(\boldsymbol{T}_{A} \equiv M g L_{0} \tilde{\boldsymbol{T}}_{A}\right)$ are computed with the following model:

$$
\tilde{\boldsymbol{F}}_{A}=\mu \tilde{V}_{A}^{2}\left[\left(C_{X 0}+C_{X \alpha} \alpha\right) \boldsymbol{i}_{B}+C_{Y \beta} \beta \boldsymbol{j}_{B}+\left(C_{Z 0}+C_{Z \alpha} \alpha\right) \boldsymbol{k}_{B}\right]
$$

$$
\begin{aligned}
\tilde{\boldsymbol{T}}_{A}= & \mu \tilde{V}_{A}^{2}\left[\epsilon_{b}\left(C_{l \beta} \beta+C_{l \tilde{p}} \tilde{p}\right) \boldsymbol{i}_{B}+\epsilon_{c}\left(C_{m 0}+C_{m \alpha} \alpha+C_{m \tilde{q}} \tilde{q}\right) \boldsymbol{j}_{B}\right. \\
& \left.+\epsilon_{b}\left(C_{n \beta} \beta+C_{n \tilde{r}} \tilde{)}\right) \boldsymbol{k}_{B}\right]
\end{aligned}
$$

in which $\mu \equiv \rho A L_{0} / 2 M, \epsilon_{b}=b / L_{0}, \epsilon_{c}=c / L_{0}, \tilde{p}=p b / 2 V_{T}$, $\tilde{q}=q c / V_{T}, \tilde{r}=r b / 2 V_{T}$, and $V_{T}$ a reference velocity. The attack and sideslip angles are

$$
\alpha=\arctan \left(\frac{\tilde{\boldsymbol{V}}_{A} \cdot \boldsymbol{k}_{B}}{\tilde{\boldsymbol{V}}_{A} \cdot \boldsymbol{i}_{B}}\right), \quad \beta=\arcsin \left(\frac{\tilde{\boldsymbol{V}}_{A} \cdot \boldsymbol{j}_{B}}{\left|\tilde{\boldsymbol{V}}_{A}\right|}\right)
$$

\section{Lagrange Equations}

The Lagrangian formulation is very appropriate to derive the equations of motion because it provides a compact system of ordinary differential equations. The constraints imposed by the bridle lines and the main tether are automatically included in the model, and the constraint force at the frictionless point $Q$ does not appear explicitly. Lagrange equations in dimensionless form read

$$
\frac{\mathrm{d}}{\mathrm{d} \tau}\left(\frac{\partial \tilde{\mathcal{L}}}{\partial \dot{x}_{s i}}\right)-\frac{\partial \tilde{\mathcal{L}}}{\partial x_{s i}}=\tilde{Q}_{i}, \quad i=1, \ldots, 5
$$

in which $\tilde{\mathcal{L}}=\tilde{E}_{k}-\tilde{\mathcal{U}}$ and the generalized forces are given by

$$
\tilde{Q}_{i}=\tilde{F}_{A} \cdot \frac{\partial \tilde{\boldsymbol{v}}_{G}}{\partial \dot{x}_{s i}}+\tilde{T}_{A} \cdot \frac{\partial \tilde{\boldsymbol{\omega}}}{\partial \dot{x}_{s i}}
$$

Because our system is holonomic, the constraint forces (i.e., the tensions in the bridle and the main tether) do not contribute to the virtual work (neither to the generalized forces $\tilde{Q}_{i}$ ).

Using Eqs. () $),(\underline{5}),(\underline{6})$, and (ㄱ) in Eq. (므) yields

$$
\begin{aligned}
& M_{s i j} \ddot{x}_{s j}+M_{s c i j} \ddot{x}_{c j}+\frac{\partial M_{s i j}}{\partial x_{s k}} \dot{x}_{s k} \dot{x}_{s j}+\frac{\partial M_{s c i j}}{\partial x_{s k}} \dot{x}_{s k} \dot{x}_{c j}+\frac{\partial M_{s i j}}{\partial x_{c k}} \dot{x}_{c k} \dot{x}_{s j} \\
& +\frac{\partial M_{s c i j}}{\partial x_{c k}} \dot{x}_{c k} \dot{x}_{c j}-\frac{1}{2}\left(\frac{\partial M_{s j k}}{\partial x_{s i}} \dot{x}_{s j} \dot{x}_{s k}+2 \frac{\partial M_{s c j k}}{\partial x_{s i}} \dot{x}_{s j} \dot{x}_{c k}+\frac{\partial M_{c j k}}{\partial x_{s i}} \dot{x}_{c j} \dot{x}_{c k}\right) \\
& +\frac{\partial \tilde{\mathcal{U}}}{\partial x_{s i}}=S_{j i} R_{j k} \tilde{F}_{A k}+\tilde{T}_{A j} \Omega_{j i}
\end{aligned}
$$

with $i=1, \ldots, 5$. Here, we used Einstein notation, that is, when an index variable appears twice in a single term, it implies summation of that term over all the values of the index. We also took into account that matrices $\overline{\boldsymbol{M}}_{s}, \overline{\boldsymbol{M}}_{s c}$, and $\overline{\boldsymbol{M}}_{c}$ depend on $\boldsymbol{x}_{s}$, but not on $\dot{\boldsymbol{x}}_{s}$. Introducing the vector $\boldsymbol{u}=\left[\begin{array}{ll}\dot{\boldsymbol{x}}_{s} & \boldsymbol{x}_{s}\end{array}\right]$, and provided that matrix $\overrightarrow{\boldsymbol{M}}_{s}$ can be inverted, system (14) takes the form of a nonautonomous system of ordinary differential equations:

$$
\frac{\mathrm{d} \boldsymbol{u}}{\mathrm{d} \tau}=\boldsymbol{f}(\boldsymbol{u}, \tau)
$$

System (14) is a completely self-contained and closed model. Given a set of initial conditions $\boldsymbol{u}_{0}$, dimensionless parameters, and control laws $\boldsymbol{x}_{c}(\tau)$, it can be integrated numerically to find the kite trajectory. Although cumbersome, it can be easily implemented in a numerical flight simulator. This implementation should be modular and constructed from the bottom to the top. For instance, one may start by writing four simple programs or modules that receive $\boldsymbol{x}_{s}$ and $\boldsymbol{x}_{c}$, and compute $\overline{\boldsymbol{\Omega}}, \overline{\boldsymbol{R}}, \overline{\boldsymbol{S}}$, and $\overline{\boldsymbol{C}}$ (see Appendix). These modules are called by other subroutines that find $\overline{\boldsymbol{M}}_{s}, \overline{\boldsymbol{M}}_{s c}$, and $\overline{\boldsymbol{M}}_{c}$. Separate subroutines for the derivatives of these tensors with respect to $\boldsymbol{x}_{s}$ and $\boldsymbol{x}_{c}$, as well as the terms $\partial \tilde{\mathcal{U}} / \partial x_{s i}, \tilde{F}_{A k}$, and $\tilde{T}_{A k}$, must also be written. Explicit expressions for all these terms are found analytically by using the results of Sec. II and Appendix. All these subroutines are called by a program that computes the right-hand side of Eq. (15), and is the core of a numerical integrator. Some tests to check the correct implementation of the simulator are given in Sec. III.

\section{Constraint Forces and Energy Balance}

Once the evolution of the state vector $\boldsymbol{x}_{s}(\tau)$ is known, by integrating system (15), the forces of constraint can be computed. In particular, the resultants of the tension forces at the attachment points $A, B, C$ are found from the equation of motion of the center of mass of the kite:

$$
\tilde{\boldsymbol{F}}_{T} \equiv \frac{\boldsymbol{F}_{T}}{M g}=\left(\frac{\mathrm{d} \tilde{\boldsymbol{v}}_{G}}{\mathrm{~d} \tau}\right)_{E}-\boldsymbol{k}_{E}-\tilde{\boldsymbol{F}}_{A}
$$

in which $\tilde{\boldsymbol{F}}_{T} \equiv \tilde{t}_{A} \boldsymbol{u}_{A}+\tilde{t}_{B} \boldsymbol{u}_{B}+\tilde{t}_{C} \boldsymbol{u}_{C}$ is the total dimensionless tension force acting on the kite, and $\boldsymbol{u}_{\nu}, \nu=A, B, C$ are the unit vectors along the bridle lines (see Appendix A2). After computing a trajectory, one may check that $\tilde{t}_{\alpha}>0$. (The bridle line is under traction.) Otherwise, the model would fail because the line would collapse. Given $\boldsymbol{u}(\tau)=\left[\boldsymbol{x}_{s}, \dot{\boldsymbol{x}}_{s}\right]$ and $\boldsymbol{x}_{c}(\tau)$, the components $\mathrm{d} \tilde{v}_{G i} / \mathrm{d} \tau$ in Eq. (16) are obtained from a simple kinematics calculation:

$$
\begin{aligned}
\frac{\mathrm{d} \tilde{v}_{G i}}{\mathrm{~d} \tau}= & S_{i j} f_{j}+\frac{\partial S_{i j}}{\partial x_{s k}} \dot{x}_{s k} \dot{x}_{s j}+\frac{\partial S_{i j}}{\partial x_{c k}} \dot{x}_{c k} \dot{x}_{s j}+C_{i j} \ddot{x}_{c}+\frac{\partial C_{i j}}{\partial x_{s k}} \dot{x}_{s k} \dot{x}_{c j} \\
& +\frac{\partial C_{i j}}{\partial x_{c k}} \dot{x}_{c k} \dot{x}_{c j}, \quad i=1,2,3
\end{aligned}
$$

Regarding the tether, Newton's third law shows that the dimensionless force at point $Q$ acting on the tether is $-\tilde{\boldsymbol{F}}_{T}$. Because the tether is taken as a rigid massless bar, the tension force is constant through it. At the ground attachment point $O$, one has $\boldsymbol{F}_{0} \equiv$ $M g \tilde{\boldsymbol{F}}_{0}=-M g \tilde{\boldsymbol{F}}_{T}$. Obviously, this force is directed along $\overleftarrow{O Q}$, and it can be written as $\tilde{\boldsymbol{F}}_{0}=\tilde{F}_{0} \overleftarrow{O Q} /|\overleftarrow{O Q}|$. This fact can be used as a first check of the correct implementation of the simulator.

The kite energy balance is found by first writing the equation for the angular momentum, $\tilde{\boldsymbol{L}}_{G} \equiv \overline{\boldsymbol{I}}_{G} \cdot \tilde{\boldsymbol{\omega}}$ :

$$
\frac{\mathrm{d} \tilde{\boldsymbol{L}}_{G}}{\mathrm{~d} \tau}=\tilde{\boldsymbol{T}}_{A}+\tilde{\boldsymbol{T}}_{T}
$$

in $\longleftarrow$ which $\quad \tilde{\boldsymbol{T}}_{T} \equiv \tilde{t}_{A}\left(\overleftarrow{G A} / L_{0}\right) \times \boldsymbol{u}_{A}+\tilde{t}_{B}\left(\overleftarrow{G B} / L_{0}\right) \times \boldsymbol{u}_{B}+$ $\tilde{t}_{C}\left(\overleftarrow{G C} / L_{0}\right) \times \boldsymbol{u}_{C}$ is the normalized moment of the tension forces about the center of mass of the kite. The sum of the scalar product of $\tilde{\boldsymbol{v}}_{G}$ with Eq. (16) and the scalar product of $\tilde{\boldsymbol{\omega}}$ with Eq. (18) yields

$$
\Delta\left(\tilde{E}_{k}+\tilde{U}\right)=\tilde{W}_{A}+\tilde{W}_{T}
$$

in which $\tilde{W}_{A}$ and $\tilde{W}_{T}$ are the normalized works of the aerodynamic force and the tensions in the bridle

$$
\tilde{W}_{\nu}=\int_{0}^{T}\left[\left(\tilde{\boldsymbol{F}}_{\nu} \cdot \tilde{\boldsymbol{v}}_{G}\right)+\left(\tilde{\boldsymbol{T}}_{\nu} \cdot \tilde{\boldsymbol{\omega}}\right)\right] \mathrm{d} t, \quad \nu=A, T
$$

For a periodic orbit, $\boldsymbol{u}(t+\tau)=\boldsymbol{u}(\tau)$, the left-hand side of Eq. (19) vanishes over one period. Equation (19) can be used as a second test of the simulator. 
During the reel-out phase of the tether, electrical energy is generated on ground. However, energy is consumed by the kite system during the reel-in phases of the tether and the bridle lines. For a periodic trajectory of (dimensionless) period $\tau_{0}$, the net dimensionless electrical power is

$$
\dot{\tilde{W}}_{\text {ele }}=\frac{1}{\tau_{0}}\left[\int_{\tau_{\text {out }}} \tilde{F}_{0} \dot{\tilde{L}} \mathrm{~d} \tau-\left|\int_{\tau_{\text {in }}} \tilde{F}_{0} \dot{\tilde{L}} d \tau+\sum_{\nu=A, B, C} \int_{\tau_{\text {in } \nu}} t_{\nu} \dot{\tilde{l}}_{\nu} \mathrm{d} \tau\right|\right]
$$

in which the integral limit $\tau_{\text {out }}\left(\tau_{\text {in }}\right)$ covers. the time with $\dot{\tilde{L}}>0$ $(\tilde{L}<0)$. Similarly, $\tau_{\text {in } \alpha}$ refers to the time with $\tilde{l}_{\alpha}<0$ (reel-in phase of the bridle line named $\alpha$ ). Here, $\tilde{l}_{\alpha}$ is the $\tau$ derivative of the length of the bridle lines, $l_{\nu} \equiv|\nu Q|$, with $\nu=A, B, C$ (see Appendix $\underline{\mathrm{A} 2}$ ).

\section{Airborne Wind Energy}

If the proposed control laws are periodic in time, $\boldsymbol{x}_{c}(t)=$ $\boldsymbol{x}_{c}(t+T)$, system $(15)$ can exhibit periodic solutions of period $T$. If stable, these trajectories are good candidates for electrical windenergy generation. Both aspects, existence and stability, are here studied with the shooting algorithm presented in [20]. It corrects the initial condition $\boldsymbol{u}_{0}$ of the periodic orbit until its error, $\boldsymbol{u}\left(\tau=\tau_{0}\right)-\boldsymbol{u}_{0} \mid$, is below certain tolerance $\left(10^{-8}\right.$ in our calculations $)$. It also provides the eigenvalues of the monodromy matrix, $\lambda_{j}$, with $j=1, \ldots, 10$. We recall that the orbit is unstable if one of these multipliers has modulus greater than one. Once an orbit is found, we checked that the tether and the bridle lines are under traction through the trajectory, and the angle of attack is always positive. We then compute $\tilde{W}_{\text {ele }}$ from Eq. (21).

The typical inertial and aerodynamic parameter values of a kite power system are shown in Table 1 . They mostly correspond to the already constructed and tested kite system at Delft University of Technology $[13,16]$. Using these values in the model presented in Appendix $\mathrm{A} 1 \mathrm{~m}$ yields $A=8.7 \mathrm{~m}^{2}, \quad h_{G}=2 \mathrm{~m}, \quad$ and $\left[I_{x x}, I_{y y}, I_{z z}\right] \approx[15.86,3.50,13.49] \mathrm{kg} \cdot \mathrm{m}^{2}$. The characteristic time, velocity, energy, and power are $\sqrt{L_{0} / g} \approx 4.51 \mathrm{~s}$, $\sqrt{g L_{0}} \approx 44.29 \mathrm{~m} / \mathrm{s}, M g L_{0} \approx 5886 \mathrm{~J}$, and $M \sqrt{L_{0} g^{3}} \approx 1300 \mathrm{~W}$. One also finds $\mu \approx 587$. We now consider a constant wind velocity equal to $14 \mathrm{~m} / \mathrm{s}$ that yields $\tilde{V}_{w}=14 / 44.29 \approx 0.316$ in Eq. (8).

\section{A. Energy Generation with Longitudinal Control}

The simplest control laws for wind-energy generation just involve a longitudinal motion of the kite. Bridle and tether control laws may produce a reel-out phase with high angles of attack and a fast reel-in phase with low angles of attack. This concept is a pumped mill with a single kite [4]. To illustrate the maneuver, we take the control vector, $x_{c}(\tau)=[\tilde{L}, \tilde{l}, \delta, \eta]$, equal to $\tilde{l}=0.05$ :

$$
\delta(\tau)=\delta_{0}+\delta_{1} \exp \left\{-\left[\tilde{\omega}_{\delta}\left(\frac{\tau}{\tau_{0}}-\phi_{0}\right)\right]^{n}\right\}
$$

and $\eta=0$. For the normalized length of the tether, $\tilde{L}(\tau)$, we approximate the sawtooth function:

$$
1+\epsilon_{L} \begin{cases}\frac{\tau}{\tau_{1}} & 0 \leq \tau \leq \tau_{1} \\ \frac{\tau_{0}-\tau}{\tau_{0}-\tau_{1}} & \tau_{1}<\tau \leq \tau_{0}\end{cases}
$$

by its first six Fourier components.

The preceding control laws yield a time-periodic control vector with period equal to $\tau_{0}, \boldsymbol{x}_{c}(\tau)=\boldsymbol{x}_{c}\left(\tau+\tau_{0}\right)$. Examples of these control laws are shown in Figs. $2 \mathrm{a}$ and $2 \mathrm{~b}$. Here, we took the values $\tau_{1}=0.85 \tau_{0}, \epsilon_{L}=0.1, \delta_{0}=7 \overline{5} \mathrm{deg}, \overline{\delta_{1}}=15 \mathrm{deg}, n=12$, and $\tilde{\omega}_{\delta}=2.5$. Periodic orbits were computed for phase shifts for the angle $\delta, \phi_{0}=0.4,0.45$, and 0.5 , and the period $\tau_{0}$ was used as a bifurcation parameter. Neither these values for the open-loop control laws, nor the environmental parameters and the properties of the kite were selected after a thorough optimization analysis. They are just characteristic values aimed at providing the performance of the model and how it can be used to estimate the power generated by the
Table 1 Kite parameters used in the simulations

\begin{tabular}{lccc}
\hline \hline Symbol & Value & Symbol & Value \\
\hline$L_{0}$ & $100 \mathrm{~m}$ & $g$ & $9.81 \mathrm{~m} / \mathrm{s}^{2}$ \\
$c$ & $1.5 \mathrm{~m}$ & $\rho_{0}$ & $1.225 \mathrm{~kg} / \mathrm{m}^{3}$ \\
$b$ & $5.8 \mathrm{~m}$ & $V_{T}$ & $7 \mathrm{~m} / \mathrm{s}$ \\
$h$ & $3.2 \mathrm{~m}$ & $M$ & $3 \mathrm{~kg}$ \\
$C_{X 0}$ & -0.065 & $C_{l \tilde{p}}$ & -0.15 \\
$C_{X \alpha}$ & 0.176 & $C_{m 0}$ & 0.133 \\
$C_{Y \beta}$ & -1.57 & $C_{m \alpha}$ & -0.763 \\
$C_{Z 0}$ & 0.116 & $C_{m \tilde{q}}$ & -0.165 \\
$C_{Z \alpha}$ & -2.97 & $C_{n \beta}$ & -0.027 \\
$C_{l \beta}$ & -0.49 & $C_{n \tilde{r}}$ & -0.002 \\
\hline \hline
\end{tabular}

kite and the stability of the orbit. Therefore, the power generated by the kite reached low values. An analysis of optimized control laws is beyond the scope of this work.

Figure 3 shows the electrical power generated by the kite, $\dot{W}_{\text {ele }}$, vs the period of the pumping cycle. The solid, dashed, and dotted lines correspond to phase shifts $\phi_{0}=0.4,0.45$, and 0.5 in Eq. (22). A maximum appears at $\phi_{p} \sim 0.45$ and period about $67 \mathrm{~s}\left(\tau_{0} \sim 15\right)$. Interestingly, this branch of solutions is also the most stable; the (dimensionless) maximum modulus of the characteristic multiplier in the studied $\tau_{0}$ range is less than $0.43,0.2$, and 0.35 for $\phi_{0}=0.4,0.45$, and 0.5 , respectively. For these three cases, the maximum tether tension happens for $\phi_{0}=0.5$, and it is about $125 \mathrm{~N}$.

Figure 4 shows the elevation angle of the tether, $\Gamma$, and the angle of attack of the kite, $\alpha$, for an orbit close to the optimum in Fig. 3 $\left(\tau_{0}=15\right.$ and $\left.\phi_{0}=45\right)$. During the traction or reel-out phase, $0 \overline{<}$ $\tau<\tau_{1}$ (see Fig. 2a), $\Gamma$ decreases, and the angle of attack is relatively high (about $15 \mathrm{deg}$ ). In the passive or reel-in phase, $\tau_{1}<\tau<\tau_{0}$, the angle of attack is small. As a result, a net electrical power is obtained.

\section{B. Energy Generation with Longitudinal and Lateral Control}

Kite performance is enhanced if the kite flies along a figure-eight path in crosswind conditions. This is achieved in the simulator by imposing the following open-loop control law for the bridle angle $\eta$ :

$$
\eta(\tau)=\eta_{0} \sin \left(\frac{2 \pi}{\tau_{0}} \tau\right)
$$

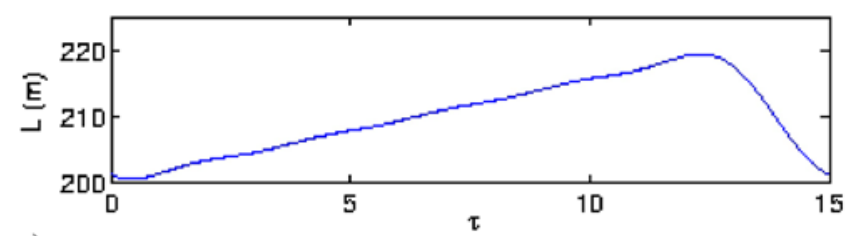

a)

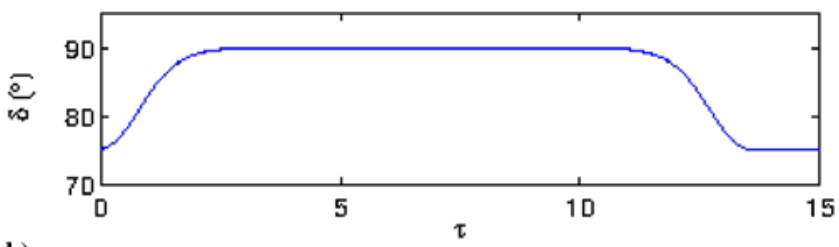

b)

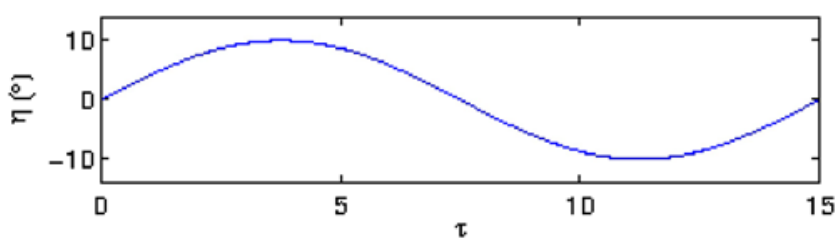

c)

Fig. 2 Example of control laws: a) tether length, and b, c) bridle angles. 
To illustrate the kite behavior under lateral control, we here consider Eq. (24), the longitudinal control laws used in Sec. IV.A with $\phi_{0}=0.45$ and $\tau_{0}=15$, and $\eta_{0}$ as a bifurcation parameter. Figure 2 shows an example of the time evolution of the tether and bridle angles $L(\tau), \delta(\tau)$, and $\eta(\tau)$ with $\eta_{0}=10 \mathrm{deg}$. Similarly to the pumped mill, the predictor-corrector algorithm was used for each value of the parameters to determine the periodic orbit and its stability. An $\eta_{0}$ family of stable periodic orbits is shown in Fig. 5. The control parameter $\eta_{0}$ was varied between 0 and $12.68 \mathrm{deg}$. For the maximum $\eta_{0}$, the lateral angle of the tether $\varphi$ reached a maximum value about $65 \mathrm{deg}$. The parameter $\eta_{0}$ was not increased beyond $12.68 \mathrm{deg}$ because the family of periodic orbit goes through a cyclic-fold bifurcation. A real Floquet characteristic multiplier approaches to +1 as $\eta_{0} \rightarrow 12.68 \mathrm{deg}$ (see Fig. 6). It suggests that a branch of unstable periodic orbits exists for $\eta_{0}<12.68 \mathrm{deg}$. The computation of this branch is beyond the scope of this work. It is also remarkable that the tension in the tether is instantaneously negative for periodic orbits within a range very close to the bifurcation point, $12.65<\eta<12.68$ (not shown). Therefore, the solutions in this $\eta_{0}$ range are not physical.

The useful electrical power $\dot{W}_{\text {ele }}$ generated by the kite system increases with the control angle of the bridle $\eta_{0}$ (see Fig. 7). For this nonoptimized case, it reaches about 300 W. Clearly, flying in crosswind conditions makes the kite more efficient from an energygeneration point of view. Unfortunately, the analysis shows that the orbit becomes less stable as the amplitudes of the figure-eight paths are enhanced (see Fig. 6).

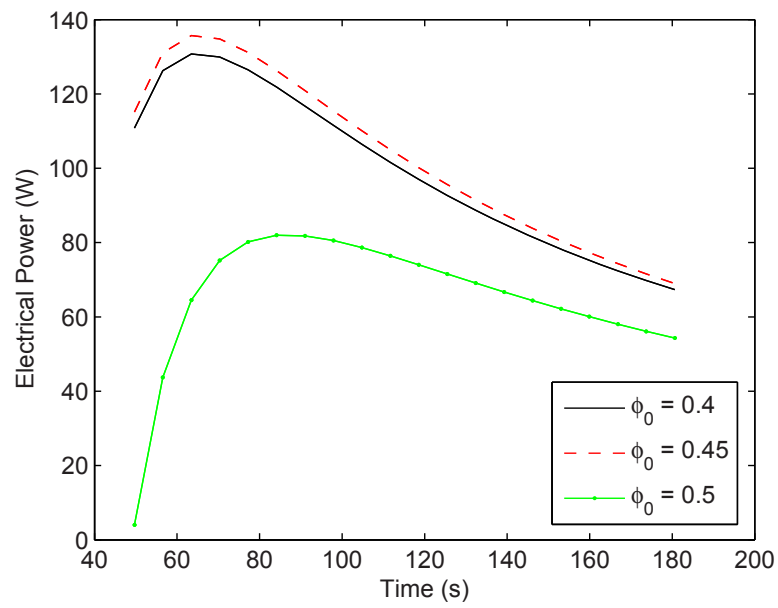

Fig. 3 Generated power vs the period for different phase shifts.

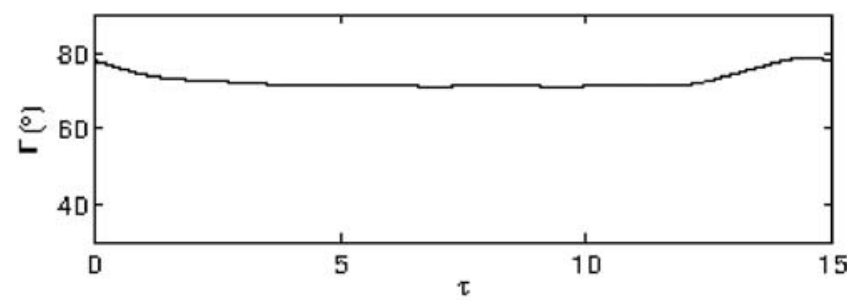

a)

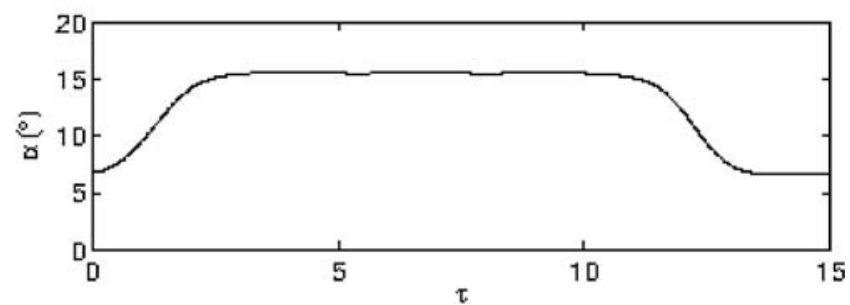

b)

Fig. 4 Evolutions of a) tether elevation angle, and b) angle of attack.

\section{Conclusions}

The implementation of airborne wind-energy systems based on pumping maneuvers needs kite flight simulators with different degrees of complexity. System design, including the properties of the kite, the bridle, the tether, and the control laws, might be found from a thorough analysis that should involve an iterative process among them. The model presented in this work has a moderate complexity. It provides the most important figures of merit of the system like generated electrical power, line tensions, kite trajectory, and stability. However, it still neglects other effects like kite flexibility or tether inertia. It extends a previous model [12] to include time-dependent bridle geometry, and it keeps some important advantages like a compact formulation and the explicit elimination of the constraint forces in the equation of motion. Equation (14), which is the main result of this work and just involves some matrices and their derivatives (see Appendix), can be easily programmed. Some tests proposed in Sec. III may help to guarantee its correct implementation.

Most of past work proposed kite and control-law designs based on the maximization of the generated power (objective function). Closed-loop controls, which require feedback and sensors, were analyzed. Here, a different strategy was followed. The pumping

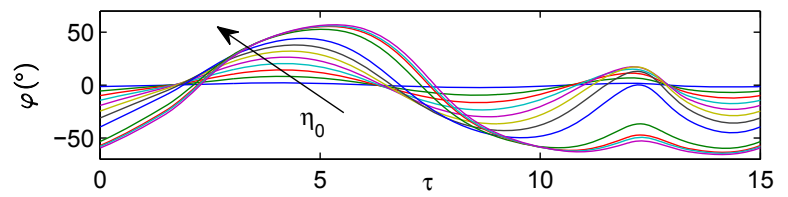

a)

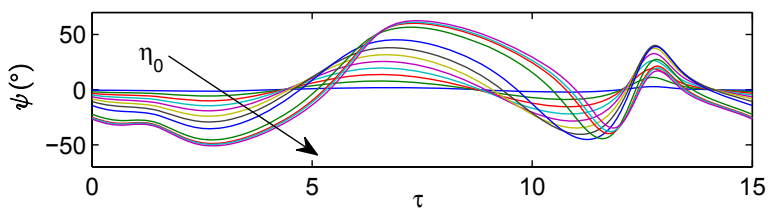

b)

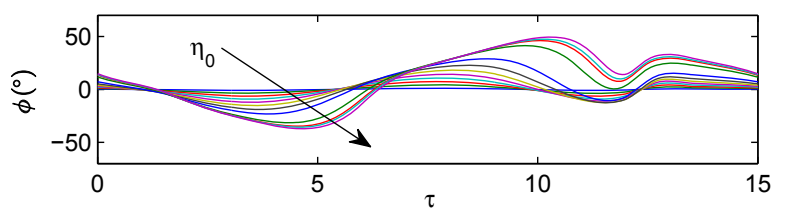

c)

Fig. 5 Periodic orbit family: a) tether angle, b) yaw, and c) roll.

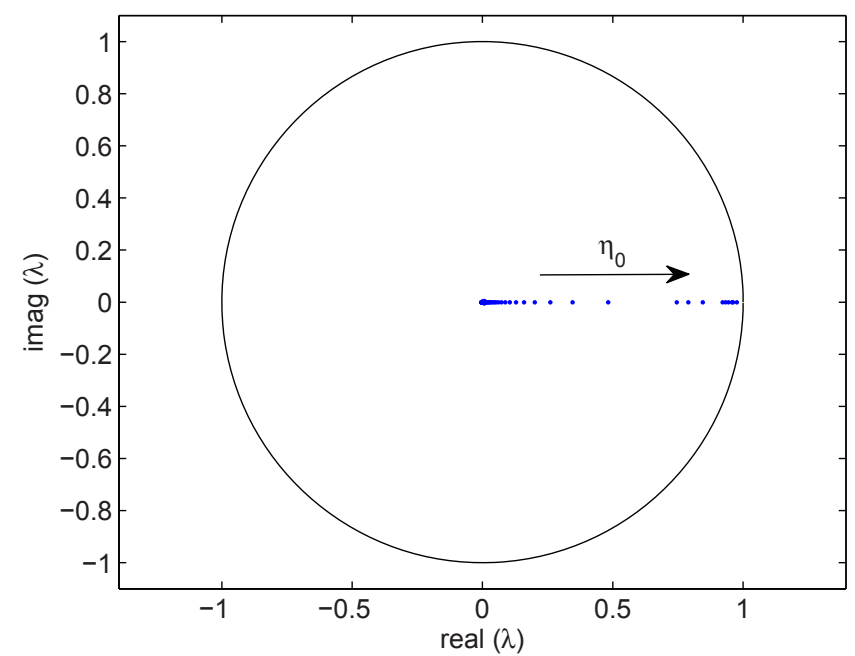

Fig. 6 Eigenvalues of the monodromy matrix for an $\eta_{0}$ family. 


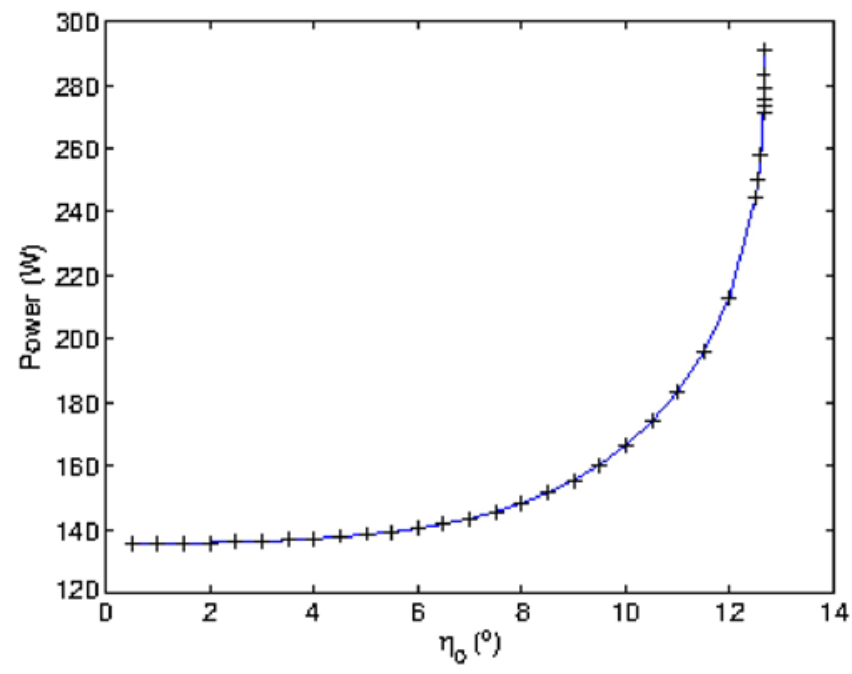

Fig. 7 Electrical power vs $\eta_{0}$.

maneuvers were placed within the framework of periodic orbits of a dynamic system, and an open-loop control was implemented. This is advantageous because stable periodic maneuvers with open-loop control do not require sensors and may yield simple and reliable operations. Even if a closed-loop control is desired, the computation of the orbit stability is interesting. In addition to the generated power, the objective functions could also contain information about the stability of the orbit (Floquet characteristic multipliers), thus improving the reliability of the system.

The results indicate that the angle $\eta_{0}$, which is determined by the lengths of the bridle lines, is very appropriate to control the kite lateral dynamics. This control could be done by an airborne kite control unit suspended at point $Q$ in Fig. 1. The simulator demonstrated that modest variations of $\eta_{0}(\sim 10 \mathrm{deg})$ yield a very high lateral displacement of the tether $(\sim 65 \mathrm{deg})$. As shown in Fig. 7, the generated electrical power also increases very rapidly with $\eta_{0}$. Therefore, parameters variation close to these values should be the subject of a deep numerical survey that may provide higher energy generation. Unfortunately, a real characteristic Floquet multiplier approaches to +1 as $\eta_{0}$ increases. It suggests that a balance between efficiency (generated energy) and reliability (stability of the orbit) should be met by the control design. A thorough analysis varying other control parameters, like the period of the orbit $\tau_{0}$ or the phase $\phi_{0}$, may help to improve both issues simultaneously.

As already mentioned, the most important effects neglected in the model are the flexibility of the kite and the inertia and flexibility of the tether. Regarding the former, a tradeoff analysis is required. Long tethers are needed to fly the kite at high altitudes, where the wind is very strong, but the dynamic behavior of the system would be severely affected. In this case, it would be necessary to adapt the simulator and solve self-consistently the partial differential equations that govern the tether dynamics. The dimension of the state vector of the simulator would be increased, and the computation of periodic orbit would become a challenging numerical problem. However, because the main advantage of the systems is, precisely, operation at high altitude, a detailed flight simulator cannot ignore it. There will be also operational constraints (such as air-traffic interaction), which should be considered in the future. A kite simulator with timedependent bridle geometry and tether dynamics will be presented in a forthcoming work.

\section{Appendix A: Auxiliary Calculations}

\section{A1 Kite Inertial Model}

Let us consider a kite of semi-elliptical cross section, semiminor axis $h$, major axis $b$, chord $c$, and constant areal density $\sigma \equiv m / A$. We take a frame of reference $S^{\prime}$ with axis parallel to frame $S_{B}$ and origin
$O^{\prime}$ in the intersection of the two planes of symmetry of the kite and the plane normal to the semi-elliptical cross section that passes through the focal points. A point of the kite is given by $\boldsymbol{r}_{p}^{\prime}\left(x^{\prime}, \theta\right)=$ $\left[b / 2(\cos \theta),-h \sin \theta, x^{\prime}\right]$ with $-c / 2 \leq x^{\prime} \leq c / 2$ and $0 \leq \theta \leq \pi$. Taking into account that the kite is a ruled surface, its area is

$$
A=\frac{b c}{2} \int_{0}^{\pi} f(\theta) \mathrm{d} \theta
$$

with $f(\theta) \equiv \sqrt{\sin ^{2} \theta+(2 h / b)^{2} \cos ^{2} \theta}$. The distance $h_{G}$ between the planes $x^{\prime} y^{\prime}$ and $x_{B} y_{B}$ is

$$
h_{G}=\left|z_{G}^{\prime}\right|=\frac{1}{m} \int\left|z_{G}^{\prime}\right| \mathrm{d} m=\frac{b c h}{2 A} \int_{0}^{\pi} \sin \theta f(\theta) \mathrm{d} \theta
$$

in which we used $\mathrm{d} m=m b f(\theta) \mathrm{d} x^{\prime} \mathrm{d} \theta / 2 A$. We then find $z_{B}=h_{G}+z^{\prime}$. In the body frame, the inertial tensor about $G, \overline{\boldsymbol{I}}_{G}$, is diagonal with elements:

$$
\begin{gathered}
I_{11}=\int\left(y_{B}^{2}+z_{B}^{2}\right) \mathrm{d} m \\
=\frac{\sigma b^{3} c}{8} \int_{0}^{\pi}\left[\cos ^{2} \theta+\left(\frac{2 h_{G}}{b}-\frac{2 h}{b} \sin \theta\right)^{2}\right] f(\theta) \mathrm{d} \theta \\
I_{22}=\int\left(x_{B}^{2}+z_{B}^{2}\right) \mathrm{d} m \\
=\frac{1}{12} m c^{2}+\frac{\sigma b c}{2} \int_{0}^{\pi}\left(h_{G}-h \sin \theta\right)^{2} f(\theta) \mathrm{d} \theta \\
I_{33}=\int\left(x_{B}^{2}+y_{B}^{2}\right) \mathrm{d} m=\frac{1}{12} m c^{2}+\frac{\sigma b^{3} c}{8} \int_{0}^{\pi} \cos ^{2} \theta f(\theta) \mathrm{d} \theta
\end{gathered}
$$

\section{A2 Bridle Lengths and Control Parameters}

The normalized length of a bridle line, say $\tilde{l}_{A} \equiv|\overleftarrow{A Q}| / L_{0}$, is found by writing $|\overleftarrow{A Q}|=|\overleftarrow{A G}+\overleftarrow{G Q}|$. Noting that $|\overleftarrow{G A}|=$ $(c / 2)\left(\boldsymbol{i}_{B}\right)+(b / 2)\left(\boldsymbol{j}_{B}\right)+h_{G} \boldsymbol{k}_{B}$, one finds

$$
\tilde{l}_{A}=\sqrt{\left(\tilde{l} c \delta c \eta-\frac{c}{2 L_{0}}\right)^{2}+\left(\tilde{l} c \delta s \eta-\frac{b}{2 L_{0}}\right)^{2}+\left(\tilde{l} s \delta-\frac{h_{G}}{L_{0}}\right)^{2}}
$$

Following a similar procedure yields

$$
\tilde{l}_{B}=\sqrt{\left(\tilde{l} c \delta c \eta-\frac{c}{2 L_{0}}\right)^{2}+\left(\tilde{l} c \delta s \eta+\frac{b}{2 L_{0}}\right)^{2}+\left(\tilde{l} s \delta-\frac{h_{G}}{L_{0}}\right)^{2}}
$$

$$
\tilde{l}_{C}=\sqrt{\left(\tilde{l} c \delta c \eta+\frac{c}{2 L_{0}}\right)^{2}+(\tilde{l} c \delta s \eta)^{2}+\left(\tilde{l} s \delta+\frac{h}{L_{0}}-\frac{h_{G}}{L_{0}}\right)^{2}}
$$

Section III used the unit vectors $\boldsymbol{u}_{A} \equiv \overleftarrow{A Q} /|\overleftarrow{A Q}|, \boldsymbol{u}_{B} \equiv \overleftarrow{B Q} /|\overleftarrow{B Q}|$ and $\boldsymbol{u}_{C} \equiv \overleftarrow{C Q} /|\overleftarrow{C Q}|$. The $\tau$ derivative of the bridle lengths as a function of the control vector and its derivative, appearing in Eq. (21), is found from Eqs. (A6-A8). 


\section{A3 Body/Earth-Frame Rotation Matrix}

Earth and body coordinates are related by $\left(x_{E}, y_{E}, z_{E}\right)^{T}=\overline{\boldsymbol{R}}\left(x_{B}, y_{B}, z_{B}\right)^{T}$, in which the rotation matrix is

$$
\boldsymbol{R}=\left(\begin{array}{ccc}
c \psi c \theta & c \psi s \theta s \phi-s \psi c \phi & c \psi s \theta c \phi+s \psi s \phi \\
s \psi c \theta & s \psi s \theta s \phi+c \psi c \phi & s \psi s \theta c \phi-c \psi s \phi \\
-s \theta & c \theta s \phi & c \theta c \phi
\end{array}\right)
$$

\section{A4 Kite Kinematics}

The angular velocity of the kite projected in the body frame is $\boldsymbol{\omega}=\overline{\boldsymbol{\Omega}} \dot{\boldsymbol{x}}_{s}$, with

$$
\overline{\mathbf{\Omega}}=\left(\begin{array}{ccccc}
0 & 0 & 0 & -s \theta & 1 \\
0 & c \phi & 0 & c \theta s \phi & 0 \\
0 & -s \phi & 0 & c \theta c \phi & 0
\end{array}\right)
$$

The velocity of the center of mass projected in the Earth frame is $\boldsymbol{v}_{G}=\overline{\boldsymbol{S}} \dot{x}_{s}+\bar{C}_{\dot{x}_{c}}$, in which

$$
\begin{aligned}
& \overline{\boldsymbol{S}}=\tilde{L} \cdot\left(\begin{array}{ccccc}
s \Gamma c \varphi & 0 & c \Gamma s \varphi & 0 & 0 \\
s \Gamma s \varphi & 0 & -c \Gamma c \varphi & 0 & 0 \\
-c \Gamma & 0 & 0 & 0 & 0
\end{array}\right) \\
& +\tilde{l} \cdot \overline{\boldsymbol{R}} \cdot\left(\begin{array}{cccc}
0-s \delta c \phi-c \delta s \eta s \phi & 0 c \theta(c \delta s \eta c \phi-s \delta s \phi) & 0 \\
0 & c \delta c \eta s \phi & 0-c \delta c \theta c \eta c \phi-s \delta s \theta & s \delta \\
0 & c \delta c \eta c \phi & 0 c \delta(s \eta s \theta+c \eta c \theta s \phi) & -c \delta s \eta
\end{array}\right)
\end{aligned}
$$

$$
\begin{aligned}
\overline{\boldsymbol{C}} & =\left(\begin{array}{cccc}
-\cos \Gamma \cos \varphi & 0 & 0 & 0 \\
-\cos \Gamma \sin \varphi & 0 & 0 & 0 \\
-\sin \Gamma & 0 & 0 & 0
\end{array}\right)+\overline{\boldsymbol{R}} \\
& \cdot\left(\begin{array}{cccc}
0 & -c \delta c \eta & \tilde{l} s \delta c \eta & \tilde{l} c \delta s \eta \\
0 & -c \delta s \eta & \tilde{l} s \delta s \eta & -\tilde{l} c \delta c \eta \\
0 & -s \delta & -\tilde{l} c \delta & 0
\end{array}\right)
\end{aligned}
$$

\section{References}

[1] Archer, C. L., and Caldeira, K., "Global Assessment of High-Altitude Wind Power," Energies, Vol. 2, No. 2, 2009, pp. 307-319. doi:10.3390/en20200307

[2] Loyd, M. L., "Crosswind Kite Power," Journal of Energy, Vol. 4, No. 3, 1980, pp. 24-30.

[3] Ockels, W. J., "Laddermill, a Novel Concept to Exploit the Energy in the Airspace," Aircraft Design, Vol. 4, No. 2, 2001, pp. 81-97. doi:10.1016/S1369-8869(01)00002-7

[4] Lansdorp, B., and Ockels, W. J., "Comparison of Concepts for HighAltitude Wind Energy Generation with Ground Based Generator," Proceedings of the Second China International Renewable Energy
Equipment and Technology Exhibition and Conference (NRE2005), Ministry of Natural Resources and Environment, Beijing, May 2005, pp. 409-417.

[5] MacCleery, B., "The Advent of Airborne Wind Power," Wind Systems Magazine, Vol. 3, No. 17, 2011, pp. 24-30.

[6] Schmehl, R., "Kiting for Wind Power," Wind Systems Magazine, Vol. 27, No. 7, 2012, pp. 36-43.

[7] Bairstow, L., The Stability of Kite Balloons, Aeronautical Research Council Repts. and Memoranda No. 208, England, U.K., 1915.

[8] Glauert, H., The Stability of a Body Towed by a Light Wire, Aeronautical Research Council Repts. and Memoranda No. 1312, England, U.K., 1930.

[9] Adomaitis, R. A., "Kites and Bifurcation Theory," SIAM Review, Vol. 31, No. 3, 1989, pp. 478-483.

[10] Sánchez-Arriaga, G., "Dynamics and Control of Single Line Kites," Aeronautical Journal, Vol. 110, No. 1111, 2006, pp. 615-621.

[11] Terink, E., Breukels, J., Schmehl, R., and Ockels, W., "Flight Dynamics and Stability of a Tethered Inflatable Kiteplane," Journal of Aircraft, Vol. 48, No. 2, 2012, pp. 503-513. doi:10.2514/1.C031108

[12] Salord Losantos, L., and Sánchez-Arriaga, G., "Flight Dynamics and Stability of Kites in Steady and Unsteady Wind Conditions," Journal of Aircraft (to be published) doi:10.2514/1.C032825

[13] de Groot, S. G. C., Breukels, J., Schmehl, R., and Ockels, W., "Modeling Kite Flight Dynamics Using a Multibody Reduction Approach," Journal of Guidance, Control, and Dynamics, Vol. 34, No. 6, 2011, pp. 1671-1682. doi: $10.2514 / 1.52686$

[14] Ilzhöfer, A., Houska, B., and Diehl, M., "Nonlinear MPC of Kites Under Varying Wind Conditions for a New Class of Large-Scale Wind Power Generators," International Journal of Robust and Nonlinear Control, Vol. 17, No. 17, 2007, pp. 1590-1599. doi:10.1002/rnc. 1210

[15] Williams, P., Lansdorp, B., and Ockels, W., "Nonlinear Control and Estimation of a Tethered Kite in Changing Wind Conditions," Journal of Guidance, Control, and Dynamics, Vol. 31, No. 3, 2008, pp. 793-799. doi:10.2514/1.31604

[16] Williams, P., Lansdorp, B., and Ockels, W., "Optimal Crosswind Towing and Power Generation with Tethered Kites," Journal of Guidance, Control, and Dynamics, Vol. 31, No. 1, 2008, pp. 81-93. doi:10.2514/1.30089

[17] Baayen, J., and Ockels, W., "Tracking Control with Adaption of Kites," IET Control Theory \& Applications, Vol. 6, No. 2, Jan. 2012, pp. 182 191. doi:10.1049/iet-cta.2011.0037

[18] Fagiano, L., Zgraggen, A., Morari, M., and Khammash, M., "Automatic Crosswind Flight of Tethered Wings for Airborne Wind Energy: Modeling, Control Design, and Experimental Results," IEEE Transactions on Control Systems Technology, Vol. 22, No. 4, July 2014 pp. 1433-1447. doi:10.1109/TCST.2013.2279592

[19] Jehle, C., and Schmehl, R., "Applied Tracking Control for Kite Power Systems," Journal of Guidance, Control, and Dynamics, Vol. 37, No. 4, 2014, pp. 1211-1222. doi: $10.2514 / 1.62380$

[20] Lara, M., and Peláez, J., "On the Numerical Continuation of Periodic Orbits: An Intrinsic, 3-Dimensional, Differential, Predictor-Corrector Algorithm," Astronomy \& Astrophysics, Vol. 389, No. 2, July 2002, pp. $692-701$ doi:10.1051/0004-6361:20020598

R. Hess Associate Editor 